\title{
ON THE DIMENSION OF VARIETIES OF SPECIAL DIVISORS
}

\author{
BY
}

R. F. LAX

\begin{abstract}
Let $T_{g}$ denote the Teichmuller space and let $V$ denote the universal family of Teichmuller surfaces of genus $g$. Let $V r_{g}^{(n)}$ denote the $n$th
\end{abstract} symmetric product of $V$ over $T_{g}$ and let $J$ denote the family of Jacobians over $T_{g}$. Let $f: V_{T_{g}}^{(n)} \rightarrow \mathrm{J}$ be the natural relativization over $T_{g}$ of the classical map defined by integrating holomorphic differentials. Let

$$
u: f^{*} \Omega_{\mathrm{J} / T_{g}}^{1} \rightarrow \Omega_{V_{T_{g} / T_{g}}^{(n)}}^{1}
$$

be the map induced by $f$. We define $G_{n}^{r}$ to be the analytic subspace of $V_{T_{g}}^{(n)}$ defined by the vanishing of $\wedge^{n-r+1} u$.

Put $\tau=(r+1)(n-r)-r g$. We show that $G_{n}^{1}-G_{n}^{2}$, if nonempty, is smooth of pure dimension $3 g-3+\tau+1$. From this result, we may conclude that, for a generic curve $X$, the fiber of $G_{n}^{1}-G_{n}^{2}$ over the module point of $X$, if nonempty, is smooth of pure dimension $\tau+1$, a classical assertion.

Variational formulas due to Schiffer and Spencer and Rauch are employed in the study of $G_{n}^{r}$

0. Introduction. Let $X$ be a complete, nonsingular curve of genus $g$ over an algebraically closed field $K$. Let $X^{(n)}$ denote the $n$th symmetric product of $X$. Let $G_{n}^{r}(X)$ denote the subvariety of $X^{(n)}$ of all divisors $D$ of degree $n$ such that $\operatorname{dim}|D| \geqslant r$. (In the literature, e.g. [12], $G_{n}^{r}(X)$ is often used to denote the subvariety of the Jacobian of $X$ consisting of all linear systerns of degree $n$ and projective dimension at least $r$.)

Put $\tau$ equal to $(r+1)(n-r)-r g$. Brill and Noether [2] asserted that if $\tau$ were nonnegative and $X$ were a generic curve, then $G_{n}^{r}(X)$ would have dimension $\tau+r$. The recent work of Kleiman and Laksov ([10], [11]) and Kempf [8] shows that for $X$ any curve, if $\tau \geqslant 0$, then $G_{n}^{r}(X)$ has dimension at least $\tau+r$. We will show, in the case $K=\mathrm{C}$, that if $X$ is a generic curve, then $G_{n}^{1}(X)-G_{n}^{2}(X)$, if nonempty, has dimension $\tau+1$.

Received by the editors December 19, 1973.

AMS (MOS) subject classifications (1970). Primary 14H15, 32G15; Secondary 14H40, $14 \mathrm{C20}$.

Key words and phrases. Analytic space, special divisor, Teichmuller surface, moduli. 
We work in the category of analytic spaces over $\mathbf{C}$. We do this because we want to consider the Teichmüller space, an analytic, but not algebraic, variety [5]. We take the Séminaire Cartan, 1960-61, as our foundational reference. In particular, we allow the structure sheaf of an analytic space to contain nilpotents.

Let $Y$ be an analytic space over $\mathrm{C}$ and let $E$ and $F$ be locally free $O_{Y^{-}}$ modules of ranks $g$ and $n$ respectively. Suppose we are given a map $u: E \rightarrow$ $F$. In $\S 1$, we define the analytic space $Z^{r}(u)$ to be given by the vanishing of the map $\bigwedge^{n-r+1} u$. We then study the infinitesimal structure of $Z^{r}(u)$.

Let $S$ be an analytic space over $\mathrm{C}$ and let $X$ be a family of nonsingular curves of genus $g$ over $S$. Let $X_{S}^{(n)}$ denote the $n$th symmetric product of $X$ over $S$ and let $J_{S}$ denote the family of Jacobians over $S$ (cf. [7], [15]). Suppose we are given a map $f: X_{S}^{(n)} \rightarrow J_{S}$. Let

$$
u: f^{*} \Omega_{\mathrm{J}_{S / S}}^{1} \rightarrow \Omega_{X_{S / S}^{(n)}}^{1}
$$

be the map induced by $f$. We study the analytic space $Z^{r}(u) \subseteq X_{S}^{(n)}$ in the following situation: $S=T_{g}$, the Teichmüller space, $X$ is the universal family of Teichmüller surfaces of genus $g$, and $f$ is the natural relativization over $T_{g}$ of the classical map from the $n$th symmetric product of a curve into its Jacobian defined by integrating a basis of homomorphic differentials (cf. $\S 2$ ). We let $G_{n}^{r}$ denote $Z^{r}(u)$ in this situation.

In order to understand explicitly the above map $f$, we must use.certain variational formulas which are similar to those derived by Schiffer and Spencer [19], but much closer in form to those appearing in Rauch [18]. We also need a theorem due to Patt [17] concerning local coordinates at a point of $T_{g}$.

Our main result is:

THEOREM. Suppose $y \in G_{n}^{1}-G_{n}^{2}$. Then the dimension of the tangent space to $G_{n}^{1}$ at $y$ is $3 g-3+\tau+1$.

From this result, we can conclude that if $X$ is a generic compact Riemann surface, then $G_{n}^{1}(X)-G_{n}^{2}(X)$, if nonempty, is smooth of pure dimension $\tau+1$.

As an application, we show that the subvariety of $T_{g}$, for $g \geqslant 4$, of curves with nonempty $G_{3}^{1}$ (so-called "trigonal" curves), is of dimension $2 g+1$, a result which was known to Severi [22] and B. Segre [20].

In a sequel to this paper, we will show that if $\tau \geqslant 0$ then $G_{n}^{2}$ (resp. $G_{n}^{3}$ ) has a component of dimension $3 g-3+\tau+2$ (resp. $3 g-3+\tau+3$ ). The proof involves computations using the examples of Riemann surfaces given by Meis [16].

The author would like to thank D. Laksov, M. Artin, A. Mattuck and, most of all, his thesis advisor S. L. Kleiman for their generous help. 
1. $Z^{r}(u)$ and its infinitesimal structure. Let $S$ be an analytic space over C. Denote by $((A n / S))$ the category of analytic spaces over $S$. Let $Y$ be an analytic space over $S$ and let $E$ and $F$ be locally free $O_{Y}$-modules of ranks $g$ and $n$ respectively. Suppose we are given a map $u: E \rightarrow F$. Define the functor $Z^{r}(u):((A n / S))^{0} \rightarrow(($ Sets $))$ by

$$
Z^{r}(u)(T)=\left\{g \in \operatorname{Hom}(T, Y) \mid \bigwedge^{n-r+1} g^{*} u=0\right\}
$$

We wish to show that this functor is represented by an analytic subspace of $Y$.

DEFINITION 1 [5]. Let $S$ be an analytic space and let

$$
G:((A n / S))^{0} \rightarrow((\text { Sets }))
$$

be a functor. We say that $G$ is of a local nature if for every $T$ the presheaf $U \mapsto G(U)$, where $U$ runs through the open sets of $T$, is a sheaf.

REMARK. This is the analog to the notion of a Zariski sheaf in the category of contravariant functors from ((Schemes)) to ((Sets)).

Lemma 1. Let $\left(S_{i}\right)$ be a covering of an analytic space $S$ by open sets. Let $G:((A n / S))^{0} \rightarrow(($ Sets $))$ be a functor. Then $G$ is representable iff $G$ is of a local nature and for every $i$, the functor $G / S_{i}:\left(\left(A n / S_{i}\right)\right)^{0} \rightarrow((\operatorname{Sets}))$ is representable.

Proof. [5, Corollary 5.7 of Exposé 7].

Our functor $Z^{r}(u)$ is clearly of a local nature. Hence, by the lemma, its representability is a local question.

Let $y$ be a point of $Y$. Since $E$ and $F$ are locally free of ranks $g$ and $n$ respectively, the map $u$ is given locally at $y$ by an $n \times g$ matrix $\left[f_{j k}\right]$ of functions regular at $y$. The functor $Z^{r}(u)$ is then locally represented by the closed analytic subspace defined by the vanishing of the minors of order $n-r+$ 1 of the matrix $\left[f_{j k}\right]$. Thus we have

Proposition 1. $Z^{r}(u)$ is represented by a closed analytic subspace of $Y$.

We will also use $Z^{r}(u)$ to denote this analytic subspace.

Put $\rho=\operatorname{rank}(u \otimes \kappa(y))$. Locally at $y$, both $E$ and $F$ split off a direct summand of rank $\rho$, and $u$ maps one summand isomorphically onto the other. The map that $u$ induces on the other two summands is given by an $(n-\rho) \times$ $(g-\rho)$ matrix $\left[e_{j k}\right]$ of functions regular at $y$. The analytic space $Z^{r}(u)$ is also defined locally at $y$ by the vanishing of the minors of order $(n-r+1-\rho)$ of the matrix $\left[e_{j k}\right]$ (cf. [10]).

Proposition 2. Assume $r>0$. Then the points of $Z^{r+1}(u)$ are singular points of $Z^{r}(u)$. 
Proof. Suppose $y \in Z^{r+1}(u)$. Then we have $\rho<n-r$. By construction, the $e_{j k}$ above vanish at $y$, hence are in the maximal ideal $m$ of $O_{Y, y}$. The analytic space $Z^{r}(u)$ is defined locally at $y$ by the vanishing of the minors of order $(n-r+1-\rho)$ of the matrix $\left[e_{j k}\right]$ and, since $\rho<n-r$, all these minors are of order at least 2 , hence are in $m^{2}$. Thus $y$ cannot be a smooth point of $Z^{r}(u)$.

We want now to study the infinitesimal structure of $Z^{r}(u)$. Let $\xi$ denote a tangent vector to $Y$ at $y$. We will also use $\xi$ to denote the comorphism, which is a $\mathrm{C}$-homomorphism of local rings $\xi: O_{Y, y} \rightarrow \mathrm{C}[\epsilon] /\left(\epsilon^{2}\right)$.

We are interested in seeing when $\xi$ is a tangent vector to $Z^{r}(u)$ at $y$. By definition, this will be true if $\bigwedge^{n-r+1} \xi^{*} u=0$.

Proposition 3. $\xi$ is a tangent vector to $Z^{r}(u)$ at $y$ iff the minors of order $n-r+1$ of the matrix $\left[\xi\left(f_{j k}\right)\right]$ are all zero.

Proof. It is easy to see that the map $\xi^{*} u$ is given by the matrix [ $\left.\xi\left(f_{j k}\right)\right]$. Thus we have $\bigwedge^{n-r+1} \xi^{*} u=0$ iff the minors of order $n-r+1$ of $\left[\xi\left(f_{j k}\right)\right]$ all vanish.

We now assume that $Y$ is smooth of dimension $m$ over C. Let $y \in Y$ and let $\sigma_{1}, \cdots, \sigma_{m}$ be local parameters on $Y$ at $y$. Let $s_{l}$ in $\mathrm{C}$ be given by

$$
\xi\left(\sigma_{l}\right)=s_{l} \epsilon, \quad l=1,2, \cdots, m .
$$

Then, by Taylor's Theorem, we have

$$
\xi\left(f_{j k}\right)=f_{j k}(y)+\epsilon \sum_{l=1}^{m} s_{l} \frac{\partial f_{j k}}{\partial \sigma_{l}}(y) .
$$

The vanishing of the minors of order $n-r+1$ of the matrix $\left[\xi\left(f_{j k}\right)\right]$ gives rise to linear equations in the $s_{l}$. These equations must be satisfied for $\xi$ to be a tangent vector to $Z^{r}(u)$ at $y$. If we view $s_{1}, \cdots, s_{m}$ as being unknowns, then the dimension of the solution space of this system of equations is the dimension of the tangent space to $Z^{r}(u)$ at $y$.

If $y \in Z^{r}(u)-Z^{r+1}(u)$, we will want to use the following lemma.

Lemma 2. Let $A$ be a commutative ring (with unit). Let $M=\left[a_{j k}\right]$ be an $m \times n$ matrix over $A$. Suppose that a minor $\mu$ of order $r$ is a unit, and that every minor of order $r+1$ containing $\mu$ vanishes. Then every minor of order $r+1$ vanishes.

Proof. Without loss of generality, we may assume that $\mu$ is the leading (i.e., upper left) minor of order $r$. Since $\mu$ is a unit, we may perform column operations using the first $r$ columns to change $M$ to the matrix 


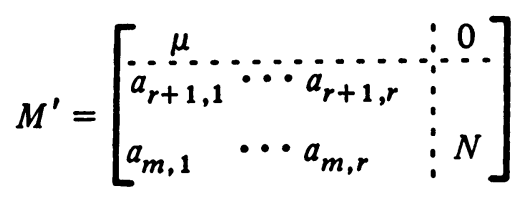

where $N$ is an $(m-r) \times(n-r)$ matrix.

Then by row operations, using the first $r$ rows, we may change $M^{\prime}$ to the matrix

$$
M^{\prime \prime}=\left[\begin{array}{ccc}
\mu & : & 0 \\
\hdashline 0 & \vdots & N
\end{array}\right]
$$

where $N$ is the same matrix as before.

Now, no minor containing $\mu$ is affected by performing these row and column operations. Hence, the minors of order $r+1$ of $M^{\prime \prime}$ which contain $\mu$ are all zero. Thus $N$ is the zero matrix.

But this implies that every column of $M$ is a linear combination of the first $r$ columns of $M$. Hence, every minor of order $r+1$ of $M$ is zero.

Suppose now that $y \in Z^{r}(u)-Z^{r+1}(u)$. Then the matrix $\left[f_{j k}\right]$ has rank $n-r$. We may thus assume that the leading minor of order $n-r$ of $\left[f_{j k}\right]$, call it $\mu$, is nonzero. Let $\mu^{\prime}$ denote the leading minor of order $n-r$ of $\left[\xi\left(f_{j k}\right)\right]$. Then $\mu^{\prime}=\mu+c \epsilon$, for some $c \in \mathbf{C}$. Since $\mu$ is nonzero, $\mu^{\prime}$ does not lie in the maximal ideal of $\mathbf{C}[\epsilon] /\left(\epsilon^{2}\right)$, hence is a unit. We then have, by Proposition 3 and Lemma 2, that $\xi$ is a tangent vector to $Z^{r}(u)$ at $y$ iff the minors of order $n-r+1$ of $\left[\xi\left(f_{j k}\right)\right]$ which contain $\mu^{\prime}$ all vanish. Obviously, there are $r(g-n+r)$ such minors. If the equations in the $s_{l}$ given by the vanishing of these minors are linearly independent (over $C$ ), then the dimension of the tangent space to $Z^{r}(u)$ at $y$ is $m-r(g-n+r)$. We could then conclude that $y$ is a smooth point of $Z^{r}(u)$ by virtue of the following proposition.

Proposition 4. Either $Z^{r}(u)$ is empty, or each component has codimension at most $r(g-n+r)$ in $Y$.

Proof. This is proved in [9] for $Y$ a scheme. With the obvious modifications, the proof is valid for $Y$ an analytic space.

2. The universal family of Teichmüller surfaces. In [5], Grothendieck proved the following

THEOREM 1. There exist an analytic space $T_{g}$ and a family $V$ of Teichmüller surfaces of genus $g$ over $T_{g}$ which is universal in the following sense: for every family $X$ of Teichmüller surfaces of genus $g$ over an analytic space $S$, there exists a unique map $\Phi: S \rightarrow T_{g}$, such that $X$ is isomorphic (as a family of Teichmïller surfaces) to the pullback via $\Phi$ of $V / T_{g}$ 
$T_{g}$ is called the Teichmüller space (for Teichmüller surfaces of genus $g$ ). The Teichmüller space is a smooth, irreducible, and simply connected analytic space [5].

Let $h: V \rightarrow T_{g}$ denote the structural morphism. By well-known topological facts, since $T_{g}$ is simply connected, the fiber bundle $R^{1} h_{*} Z$ is trivial. Thus, there are sections of this bundle which give rise to cycles $\gamma_{i}(s), \delta_{i}(s), i=1, \cdots, g$, which form a canonical homology basis for $H_{1}\left(V_{s}, \mathrm{Z}\right), s \in T_{g}$ [15].

Consider the sheaf $\Omega_{V / T_{g}}^{1}$. For all $s \in T_{g}$, we have

$$
\operatorname{dim} H^{0}\left(V_{s}, \Omega_{V / T_{g}}^{1} \otimes \kappa(s)\right)=\operatorname{dim} H^{0}\left(V_{s}, \Omega_{V_{s}}^{1}\right)=g .
$$

Hence, $h_{*} \Omega_{V / T_{g}}^{1}$ is a vector bundle of rank $g$ over $T_{g}$ and we have

$$
h_{*} \Omega_{V / T_{g}}^{1} \otimes k(s) \cong H^{0}\left(V_{s}, 2_{V_{s}}^{1}\right)
$$

by [4].

Choose holomorphic sections $d \zeta_{i}^{*}, i=1, \cdots, g$, of $h_{*} \Omega_{V / T_{g}}^{1}$ such that $\left\{d \zeta_{i}^{*}(s)\right\}_{i=1}^{g}$ is a basis for $H^{0}\left(V_{s}, \Omega_{V_{s}}^{1}\right), s \in T_{g}$ (cf. [15]). Put

$$
a_{i j}(s)=\int_{\gamma_{i}(s)} d \zeta_{j}^{*}(s), \quad b_{i j}(s)=\int_{\delta_{i}(s)} d \zeta_{j}^{*}(s), \quad i, j=1, \cdots, g .
$$

For each $s \in T_{g}$, the matrix $\left[a_{i j}(s), b_{i j}(s)\right]$ is the period matrix of $V_{s}$. Recall that the columns of this matrix generate a maximal lattice subgroup of $\mathbf{C}^{\boldsymbol{g}}$. Let $J$ be the quotient of $T_{g} \times C^{g}$ by this family of lattices. The induced projection $J \rightarrow T_{g}$ gives a complex analytic family of complex tori, the fiber $J_{s}$ being the Jacobian variety of the Teichmüller surface $V_{s}$ [15].

Since our concern will only be local, we assume that there exist sections of $V \rightarrow T_{g}$. Let $P_{0}^{*}(s)$ be such a section. As in [15], define a map $\psi: V \rightarrow J$ by

$$
\psi(s, P)=\left(s, \int_{P_{0}^{*}(s)}^{P} d \zeta_{1}^{*}(s), \cdots, \int_{P_{0}^{*}(s)}^{P} d \xi_{g}^{*}(s)\right) \bmod \text { periods }
$$

for $P \in V_{s}$.

Denote by $V_{T_{g}}^{(n)}$ the $n$th symmetric product of $V$ over $T_{g}$ (cf. [7]). Extend $\psi$ to a map $f: V_{T_{g}^{(n)}} \rightarrow J$ as follows. If $s \in T_{g}$ and $D \in\left(V_{T_{g}}^{(n)}\right)_{s}$ is the divisor $\Sigma_{i=1}^{n} P_{i}$ on $V_{s}^{8}$, then

$$
f(s, D)=\left(s, \sum_{i=1}^{n} \int_{P_{0}^{*}(s)}^{P_{i}} d \zeta_{1}^{*}(s), \cdots, \sum_{i=1}^{n} \int_{P_{0}^{*}(s)}^{P_{i}} d \zeta_{g}^{*}(s)\right) \bmod \text { periods. }
$$




$$
u: f^{*} \Omega_{\mathrm{J} / T_{g}}^{1} \rightarrow \Omega_{V_{T_{g}}^{(n)} / T_{g}}^{1}
$$

be the map induced by $f$. Since $J$ and $V_{T_{g}}^{(n)}$ are smooth over $T_{g}$ of relative dimensions $g$ and $n$ respectively, the sheaves

$$
f^{*} \Omega_{\mathrm{J} / T_{g}}^{1} \text { and } \Omega_{V_{T_{g} / T_{g}}^{(n)}}^{1}
$$

are locally free of ranks $g$ and $n$ respectively. Thus, we may consider the analytic subspace $Z^{r}(u) \subseteq V_{T_{g}}^{(n)}$ of $\S 1$. We will denote by $G_{n}^{r}$ the analytic space $Z^{r}(u)$ which arises in this situation. We will see in $\S 4$ that $\left(G_{n}^{r}\right)_{s}$ is what was denoted by $G_{n}^{r}\left(V_{s}\right)$ in $\S 0$.

We wish to study the infinitesimal structure of $G_{n}^{r}$. To do this, we need explicit knowledge of the above map $f$. And to obtain this knowledge, we need certain variational formulas which are contained in the next section.

3. The variational formula. For a detailed treatment of the material in this section, the reader is referred to Rauch [18] or Patt [17].

Let $X$ be a compact Riemann surface of genus $g>0$. Let $\Gamma=\left(\gamma_{1}, \cdots\right.$, $\left.\gamma_{g}\right)$ and $\Delta=\left(\delta_{1}, \cdots, \delta_{g}\right)$ be a canonical homotopy basis and let $\Pi$ be the simply connected surface obtained by the canonical dissection of $X$ determined by $\Gamma$ and $\Delta$ (cf. [23]).

Let $w$ be a point in the interior of $\Pi$ and let $\tau_{w, \nu}(z)$ denote the (normalized) elementary integral of the second kind with pole of order $\nu+1$ at $w$ and zero $\Gamma$-periods.

Let $\zeta$ be an Abelian integral of the first kind. Let $a_{i}, i=1, \cdots, g$, denote the $\Gamma$-periods of $d \zeta$; that is,

$$
a_{i}=\int_{\gamma_{i}} d \zeta, \quad i=1, \cdots, g .
$$

The value of the derivatives of a determination of $\zeta$ at $w$ and the periods of the differentials $d \tau_{w, \nu}$ are related by

$$
\zeta^{(\nu+1)}(w)=\frac{\nu !}{2 \pi i} \sum_{j=1}^{g} a_{j} \int_{\delta_{j}} d \tau_{w, \nu}(z)
$$

which follows from the bilinear relation for differentials of the first and second kinds $[23$, p. 260$]$.

Let $Q_{1}, \cdots, Q_{n}$ be distinct points in the interior of $\Pi$ such that all the $Q_{j}$ are different from $w$ and none of the $Q_{j}$ is a zero of $d \zeta$. Let $t_{j}, j=1$, $\cdots, n$, be a local parameter at $Q_{j}$. Let $D_{1}, \cdots, D_{n}$ be disjoint disks about $Q_{1}, \cdots, Q_{n}$ respectively, such that $D_{j}$ lies in the domain of $t_{j}$, is completely 
contained in the interior of $\Pi$ and such that no $D_{j}$ contains either $w$ or any zero of $d \zeta$. given by

Inside $D_{j}$, we can vary the local parameter $t_{j}$ to a new parameter $t_{j}^{*}$

$$
t_{j}^{*}=t_{j}+c_{j} / t_{j}, \quad j=1, \cdots, n,
$$

where $c_{j}$ is sufficiently small. This defines a new Riemann surface $X^{*}$, having the same canonical homotopy basis as $X$ has (since all variations take place in the interior of $\Pi$ ).

Let $\zeta^{*}$ be the Abelian integral of first kind on $X^{*}$ with the same $\Gamma$. periods as $\zeta$. We wish to compute

$$
\Delta \zeta^{(\nu+1)}(w)=\zeta^{*(\nu+1)}(w)-\zeta^{(\nu+1)}(w) .
$$

Notation. $d \tau_{w, \nu} d \zeta$ is a (not necessarily finite) quadratic differential on $X$. Locally at $Q_{j}$, we may write $d \tau_{w, \nu} d \zeta=h\left(t_{j}\right) d t_{j}^{2}$. We now introduce the notation $\tau_{w, \nu}^{\prime}\left(Q_{j}\right) \xi^{\prime}\left(Q_{j}\right)$ for $h(0)$.

Utilizing the techniques and formulas in [18] and [17], one can obtain the following proposition:

Proposition 5.

$$
\Delta \zeta^{(\nu+1)}(w)=\nu ! \sum_{m=1}^{n} c_{m} \tau_{w, \nu}^{\prime}\left(Q_{m}\right) \xi^{\prime}\left(Q_{m}\right)+O\left(c^{2}\right)
$$

where $c=\max _{1<m<n}\left|c_{m}\right|$.

We will also want to use the following theorem, due to Patt [17]:

TheOREM 2. One may choose $3 g-3$ points $Q_{1}, \cdots, Q_{3 g-3}$ on $X$ such that, if $c_{m}$ is the variation parameter at $Q_{m}$, then a neighborhood of the origin in the $c_{1}, \cdots, c_{3 g-3}$ space describes a complex-analytic structure for a neighborhood of $X$ in the Teichmüller space. Moreover, the set of collections of $3 g-3$ points with this property is open in $X^{3 g-3}$.

Proof. The first assertion follows from Theorems 2 and 4 of [17]. Although Patt does not state the second assertion, his proofs demonstrate it, as was noted by Farkas $[3$, p. 885$]$.

4. The equations which define the tangent space. Let $X$ be a compact Riemann surface of genus $g>1$. Let $\left\{\gamma_{j}, \delta_{j}\right\}_{j=1}^{g}$ be a canonical homotopy basis and let $\left\{d \zeta_{k}\right\}_{k=1}^{g}$ be a basis of the holomorphic differentials. Put

$$
A_{j k}=\int_{\gamma_{j}} d \zeta_{k}, \quad j, k=1, \cdots, g .
$$


Let $P$ be a point of $X$ and let $t$ be a local parameter on $X$ at $P$. Write

$$
d \zeta_{k}=\sum_{l=0}^{\infty} a_{k, l} t^{l} d t
$$

Fix a point $P_{0}$ different from $P$. Choose a point $\left(Q_{1}, \cdots, Q_{3 g-3}\right)$ from the open subset of $X^{38-3}$ in Theorem 2 such that all the $Q_{m}$ are different from $P$ and $P_{0}$ and such that none of the $Q_{m}$ is a zero of any $d \zeta_{k}$. Perform the variation described in $\S 3$, taking the disk about each $Q_{m}$ sufficiently small so that no two disks intersect and no disk contains $P, P_{0}$, or any zero of any $d \zeta_{k}$. Let $c_{m}$ denote the variation parameter at $Q_{m}, m=1, \cdots, 3 g-3$, as in $\S 3$.

Let $s_{0} \in T_{g}$ be the module point of $X$ (i.e., $V_{s_{0}}=X$ ). By definition of the variation, there exists a complex-analytic neighborhood $U$ of $s_{0}$ in $T_{g}$ such that, for all $s^{\prime} \in U$, the curves $\left\{\gamma_{j}, \delta_{j}\right\}_{j=1}^{g}$ are a canonical homotopy basis on $V_{s^{\prime}}$, the points $P_{0}$ and $P$ are on $V_{s^{\prime}}$, and $t$ is a local parameter on $V_{s^{\prime}}$ at $P$. Choose holomorphic sections $d \zeta_{k}^{*}, k=1, \cdots, g$, of $h_{*} \Omega_{V / T_{g}}^{1}$ such that

$$
\int_{\gamma_{j}} d \xi_{k}^{*}\left(s^{\prime}\right)=A_{j k}, \quad s^{\prime} \in U, \quad j, k=1, \cdots, g
$$

(cf. $[15, \S 3])$.

Proposition 6. With notation as in $\S 3$ and above, if we define $a_{k, l}^{*}$ by $d \zeta_{k}^{*}=\Sigma_{l=0}^{\infty} a_{k, l^{l}}^{*} d t$, then we have

$$
a_{k, l}^{*}=a_{k, l}+\sum_{m=1}^{3 g-3} c_{m} \tau_{P, l}^{\prime}\left(Q_{m}\right) \zeta_{k}^{\prime}\left(Q_{m}\right)+O\left(c^{2}\right) .
$$

Proof. The variational formula (Proposition 5) shows that this equality holds in a complex-analytic neighborhood of $\left(s_{0}, P\right)$ on $V$. This is the main import of the variational formula.

In order to study the map

$$
u: f^{*} \Omega_{\mathrm{J} / T_{g}}^{1} \rightarrow \Omega_{V_{T_{g} / T_{g}}^{(n)}}
$$

of $\S 2$, we first consider the divisor $n P$ on $X$. Let $t_{1}, \cdots, t_{n}$ be $n$ copies of $t$, and let $\sigma_{1}, \cdots, \sigma_{n}$ denote the $n$ elementary symmetric functions in $t_{1}, \cdots, t_{n}$.

Proposition 7. Local parameters on $V_{T_{g}}^{(n)}$ at $\left(s_{0}, n P\right)$ are given by $c_{1}, \cdots, c_{3 g-3}, \sigma_{1}, \cdots, \sigma_{n}$. 
Proof. By Theorem 2, local parameters on $T_{g}$ at $s_{0}$ are given by $c_{1}$, $\cdots, c_{3 g-3}$. By [1], local parameters on $X^{(n)}$ at $n P$ are given by $\sigma_{1}, \cdots$, $\sigma_{n}$. By the definition of the variation in $\S 3$, local parameters on $\left(V_{T_{g}}^{(n)}\right)_{s^{\prime}}$ at $n P$, for $s^{\prime} \in U$, are also given by $\sigma_{1}, \cdots, \sigma_{n}$. Thus, local parameters on $V_{T_{g}}^{(n)}$ at $\left(s_{0}, n P\right)$ are given by $c_{1}, \cdots, c_{3 g-3}, \sigma_{1}, \cdots, \sigma_{n}$.

Put

$$
\tau_{j}=t_{1}^{j} d t_{1}+\cdots+t_{n}^{j} d t_{n}, \quad j=0,1,2, \cdots .
$$

We have

PROPOSITION 8. The space of holomorphic 1-forms on $X$ is naturally isomorphic to the space of holomorphic 1-forms on $X^{(n)}$. Both these spaces are isomorphic to the space of symmetric holomorphic 1-forms on the Cartesian product $X^{n}$. If $d \xi=\sum_{l=0}^{\infty} a_{l} t^{t} d t$ is a holomorphic 1-form on $X$ and $d \tilde{\zeta}$ is the corresponding symmetric holomorphic 1-form on $X^{n}$, then $d \tilde{\zeta}=\Sigma_{l=0}^{\infty} a_{l} \tau_{l}$.

Proof. [14, pp. 226-227].

This result is easily seen to relativize to the following proposition.

PROPOSITION 9. The space of relative holomorphic 1-forms on $V_{T_{g}}^{(n)}$ over $T_{g}$ and the space of relative holomorphic 1-forms on $V$ over $T_{g}$ are naturally isomorphic. Both spaces are isomorphic to the space of relative symmetric holomorphic 1-forms on $V_{T_{g}}^{n}$, the product over $T_{g}$ of $n$ copies of $V$, over $T_{g}$. If $d \widetilde{\zeta}_{k}^{*}$ is the relative symmetric holomorphic 1 -form on $V_{T_{g}}^{n}$ over $T_{g}$ corresponding to $d \zeta_{k}^{*}$ (cf. Proposition 6), then

$$
d \tilde{\zeta}_{k}^{*}=\sum_{l=0}^{\infty} a_{k, l}^{*} \tau_{l}
$$

We will identify relative symmetric holomorphic 1-forms on $V_{T_{g}}^{n}$ over $T_{g}$ and relative holomorphic 1 -forms on $V_{T_{g}}^{(n)}$ over $T_{g}$.

Now, we can express $d \tilde{\zeta}^{*}$ in terms of $d \sigma_{1}, \cdots, d \sigma_{n}$ by using the following identities [14]:

$$
\sigma_{k} \tau_{0}-\sigma_{k-1} \tau_{1}+\cdots+(-1)^{k} \tau_{k}=d \sigma_{k+1}
$$

(By convention, $\sigma_{k}=0$ and $d \sigma_{k}=0$ if $k>n$.) Inverting these identities, and writing out only the linear terms, we obtain

$$
\begin{aligned}
\tau_{k}= & (-1)^{k}\left(d \sigma_{k+1}-\sigma_{1} d \sigma_{k}-\cdots-\sigma_{k} d \sigma_{1}\right) \\
& + \text { higher order terms. }
\end{aligned}
$$

Thus we may write 


$$
\begin{aligned}
d \tilde{\zeta}_{k}^{*}=\sum_{l=0}^{\infty}(-1)^{l}\left[\left(a_{k, l}+\sum_{m=1}^{3 g-3} c_{m} \tau_{P, l}^{\prime}\left(Q_{m}\right) \zeta_{k}^{\prime}\left(Q_{m}\right)\right)\right. \\
\left.\left(d \sigma_{l+1}-\sigma_{1} d \sigma_{l}-\cdots-\sigma_{l} d \sigma_{1}\right)\right]+O\left(\sigma^{2}, c^{2}\right)
\end{aligned}
$$

where $O\left(\sigma^{2}, c^{2}\right)$ denotes higher order terms in the $\sigma_{j}$ and the $c_{m}$.

By definition of the map $f: V_{T_{g}}^{(n)} \rightarrow J$ in $\S 2$, it is easy to see that $f$ is given at $\left(s_{0}, n P\right)$ by

$$
f\left(s_{0}, n P\right)=\left(s_{0}, \int_{P_{0}}^{P} d \tilde{\zeta}_{1}^{*}\left(s_{0}\right), \cdots, \int_{P_{0}}^{P} d \widetilde{\zeta}_{g}^{*}\left(s_{0}\right)\right) \bmod \text { periods }
$$

where the integrals $\int_{P_{0}}^{P} d \tilde{\zeta}_{k}^{*}\left(s_{0}\right)$ are evaluated by recalling that $t_{1}, \cdots, t_{n}$ are just copies of $t$. Let $\partial \widetilde{\zeta}_{k}^{*} / \partial \sigma_{j}$ be given by

$$
d \tilde{\zeta}_{k}^{*}=\sum_{j=1}^{n} \frac{\partial \tilde{\zeta}_{k}^{*}}{\partial \sigma_{j}} d \sigma_{j}
$$

Then we have

Proposition 10. The map

$$
u: f^{*} \Omega_{J / T_{g}}^{1} \rightarrow \Omega_{T_{g}^{(n)} / T_{g}}^{1}
$$

is given locally at $\left(s_{0}, n P\right)$ by the matrix

$$
\left[\partial \tilde{\zeta}_{k}^{*} / \partial \sigma_{j}\right], \quad j=1, \cdots, n, k=1, \cdots, g .
$$

Proof. This follows easily from the definitions of $f$ and $\partial \widetilde{\zeta}_{k}^{*} / \partial \sigma_{j}$. (Compare with [3] and [6].)

Remark. Let $J$ denote the Jacobian variety of $X$ and let $f_{0}: X^{(n)} \rightarrow$ $J$ be the classical map (i.e., the map $f \otimes K\left(s_{0}\right)$ ). Then the matrix $M=$ $\left[\left(\partial \tilde{\zeta}_{k}^{*} / \partial \sigma_{j}\right)\left(s_{0}, n P\right)\right]$ is the matrix of the map $u_{0}: f_{0}^{*} \Omega_{J}^{1} \rightarrow \Omega_{X}^{1}(n)$ at $n P$ (cf. [3], [6]). It is then easy to see that $\left(G_{n}^{r}\right)_{s_{0}}$ is what was denoted by $G_{n}^{r}(X)$ in $\S 0$.

Now let $\xi$ be a tangent vector to $V_{T_{g}}^{(n)}$ at $\left(s_{0}, n P\right)$. Let $s_{j}$ and $b_{m}$ in C be given by

$$
\begin{aligned}
& \xi\left(\sigma_{j}\right)=s_{j} \epsilon, \quad j=1, \cdots, n, \\
& \xi\left(c_{m}\right)=b_{m} \epsilon, \quad m=1, \cdots, 3 g-3 \text {. }
\end{aligned}
$$

Then, using Taylor's Theorem as in $\S 1$, we have 


$$
\begin{gathered}
\xi\left(\frac{\partial \tilde{\zeta}_{k}^{*}}{\partial \sigma_{j}}\right)=\frac{\partial \tilde{\zeta}_{k}^{*}}{\partial \sigma_{j}}\left(s_{0}, n P\right)+\epsilon \sum_{l=1}^{n} s_{l} \frac{\partial^{2} \tilde{\zeta}_{k}^{*}}{\partial \sigma_{l} \partial \sigma_{j}}\left(s_{0}, n P\right) \\
+\epsilon \sum_{m=1}^{3 g-3} b_{m} \frac{\partial^{2} \widetilde{\zeta}_{k}^{*}}{\partial c_{m} \partial \sigma_{j}}\left(s_{0}, n P\right) .
\end{gathered}
$$

We will now use (\#) to compute the partial derivatives of $\partial \tilde{\zeta}_{k}^{*} / \partial \sigma_{j}$ with respect to $\sigma_{l}$ and with respect to $c_{m}$. (We remind the reader that the functions $\sigma_{l}$ and $c_{m}$ vanish at $\left(s_{0}, n P\right)$.) We obtain

and

$$
\frac{\partial^{2} \widetilde{\zeta}_{k}^{*}}{\partial \sigma_{l} \partial \sigma_{j}}\left(s_{0}, n P\right)=(-1)^{j+l} a_{k, j+l-1}
$$

$$
\frac{\partial^{2} \widetilde{\zeta}_{k}^{*}}{\partial \sigma_{m} \partial \sigma_{j}}\left(s_{0}, n P\right)=\tau_{P, j-1}^{\prime}\left(Q_{m}\right) \zeta_{k}^{\prime}\left(Q_{m}\right)
$$

Substituting these expressions for the partial derivatives evaluated at $\left(s_{0}, n P\right)$ into (1) gives us

Proposition 11.

$$
\begin{aligned}
\xi\left(\frac{\partial \tilde{\zeta}_{k}^{*}}{\partial \sigma_{j}}\right)= & \frac{\partial \tilde{\zeta}_{k}^{*}}{\partial \sigma_{j}}\left(s_{0}, n P\right)+\epsilon \sum_{l=1}^{n}(-1)^{l} s_{l} a_{k, j+l-1} \\
& +\epsilon \sum_{m=1}^{3 g-3} b_{m} \tau_{P, j-1}^{\prime}\left(Q_{m}\right) \xi_{k}^{\prime}\left(Q_{m}\right) .
\end{aligned}
$$

Now on to the general case. Consider a divisor $D$ on $X$ of the form $D=m_{1} P_{1}+\cdots+m_{d} P_{d}$. Assume $D$ is in $G_{n}^{r}(X)$ and choose a basis $\left\{d \zeta_{k}\right\}_{k=1}^{g}$ of the holomorphic differentials on $X$ such that the last $i=\operatorname{dim} H^{1}\left(X, O_{X}(D)\right)$ of them vanish on $D$.

In performing the variation in $\S 3$, choose a point $\left(Q_{1}, \cdots, Q_{3 g-3}\right)$ from the open set in $X^{3 g-3}$ in Theorem 2 so that each $Q_{m}$ is different from $P_{0}$, $P_{1}, \cdots, P_{d}$ and any other zero of any $d \zeta_{k}$. (The choice of this point will be further modified later.) Take the disk about each $Q_{m}$ sufficiently smali so that no two disks intersect and such that no disk contains $P_{0}, P_{1}, \cdots, P_{d}$ or any other zero of any $d \zeta_{k}$.

Let $f_{j}: V_{T_{g}}^{\left(m_{j}\right)} \rightarrow J$ be the map defined in $\S 2$ and let

$$
u_{j}: f_{j}^{*} \Omega_{\mathrm{J} / T_{g}}^{1} \rightarrow \Omega_{V_{g} / T_{g}}^{1}\left(m_{j}\right)
$$


be the map induced by $f_{j}$. The obvious map

$$
V_{T_{g}}^{\left(m_{1}\right)} \times_{T_{g}} V_{T_{g}}^{\left(m_{2}\right)} \times_{T_{g}} \cdots \times_{T_{g}} V_{T_{g}}^{\left(m_{d}\right)} \rightarrow V_{T_{g}}^{(n)}
$$

is a local analytic isomorphism by an argument analogous to that given in [14] in the case of a curve over a field. Locally, the map $f$ is the one induced by the $f_{j}$ and the map $u$ is the one induced by the $u_{j}$. Thus, the matrix of $u$ locally at $\left(s_{0}, D\right)$ is obtained by "stacking" the matrices of the $u_{j}$ locally at $\left(s_{0}, m_{j} P_{j}\right)$.

Let $\xi$ be a tangent vector to $V_{T_{g}}^{(n)}$ at $\left(s_{0}, D\right)$ and let $\xi_{j}$ be the tangent vector to $V_{T}^{\left(m_{j}\right)}$ at $\left(s_{0}, m_{j} P_{j}\right)$ induced by $\xi$, for $j=1, \cdots, d$. Then the matrix of $\xi^{*} u$ is obtained by "stacking" the matrices of the $\xi_{j}^{*} u_{j}$, for $j=1$, $\cdots, d$.

Let $M^{\prime}$ denote the matrix of $\xi^{*} u$. Let $\mu$ denote the leading minor of order $n-r$ of $M$, the matrix $\left[\left(\partial \widetilde{\zeta}_{k}^{*} / \partial \sigma_{j}\right)\left(s_{0}, D\right)\right]$, and let $\mu^{\prime}$ denote the leading minor of order $n-r$ of $M^{\prime}$. Then we have $\mu^{\prime}=\mu+c \epsilon$ for some $c$ in $\mathbf{C}$. Now, by our choice of a basis of the holomorphic differentials on $X$, the last $i$ columns of $M$ are identically zero, hence the last $i$ columns of $M^{\prime}$ contain "pure" $\epsilon$ terms (i.e., members of the maximal ideal of $\mathbf{C}[\epsilon] /\left(\epsilon^{2}\right)$ ). Thus, in computing a minor of order $n-r+1$ containing $\mu^{\prime}$, any $\epsilon$ 's in the first $n-$ $r$ columns will be "killed" by the $\epsilon$ in the last column of the minor of order $n-r+1$. Hence, we have established

Lemma 3. For purposes of computing the minors of order $n-r+1$ of $M^{\prime}$, we may replace the first $n-r$ columns of $M^{\prime}$ by the first $n-r$ columns of $M$.

Let $M$ denote the resulting matrix.

$M$ has a particularly nice form in the case that $D=P_{1}+P_{2}+\cdots+P_{n}$, with all points distinct. Let $t_{j}$ be a local parameter at $P_{j}$ and write $d \zeta_{k}=$ $\varphi_{j, k} d t_{j}$. Then we have

$$
M=\left[\begin{array}{lll}
\varphi_{j, k}\left(P_{j}\right) & \left.\vdots \epsilon \in s_{j} \varphi_{j, k}^{\prime}\left(P_{j}\right)+\sum_{m=1}^{3 g-3} b_{m} \tau_{P_{j}, 0}^{\prime}\left(Q_{m}\right) \zeta_{k}^{\prime}\left(Q_{m}\right)\right) \\
j=1, \cdots, n & \vdots & j=1, \cdots, n \\
k=1, \cdots, g-i & k=g-i+1, \cdots, g
\end{array}\right]
$$

Going back to the general case, recall that, by Proposition 1, $\xi$ will be a tangent vector to $G_{n}^{r}$ at $\left(s_{0}, D\right)$ iff the minors of order $n-r+1$ of the matrix $M$ all vanish. Assume $D=m_{1} P_{1}+\cdots+m_{d} P_{d}$ is in $G_{n}^{r}(X)-G_{n}^{r+1}(X)$. Then the matrix $M$ has rank precisely $n-r$. Hence, by permuting the rows of 
$M$, if necessary, we end up with a matrix whose leading minor of order $n-r$, which we will denote by $\mu$, is nonzero. We will continue to denote this matrix by $M$, although its form may differ slightly from that specified earlier.

Perform the same row permutations as above on the matrix $M$ and denote the resulting matrix also by $M$. Then $\mu$ is also the leading minor of order $n-r$ of $M$, so we may apply Lemma 2. Thus, for all the minors of order $n-r+1$ of $M$ to vanish, it is sufficient that every minor of order $n-r+1$ which contains $\mu$ vanishes. The vanishing of each of these minors gives rise to a linear equation in the $s_{j}$ and the $b_{m}$.

Let $\mu_{j, k}$ denote the minor of order $n-r+1$ of $M$ obtained by adjoining to $\mu$ the first $n-r$ elements of the $(n-r+j)$ th row of $M$ and the first $n-r$ elements and the $(n-r+j)$ th element of the $(n-r+k)$ th column of $M$ (thus $j$ runs from 1 through $r$ and $k$ runs from 1 through $i$ ). The equation $\mu_{j, k}=0$ is of the form $\epsilon E_{j, k}=0$ where $E_{j, k}$ is a linear equation in the $s_{j}$ and the $b_{m}$ with coefficients in $\mathbf{C}$.

We will now view the $s_{j}$ and the $b_{m}$ as being unknowns (as in $\S 1$ ). Thus, $E_{j, k}$ is an equation in $3 g-3+n$ unknowns. By the discussion after Proposition 1 , the dimension of the tangent space to $G_{n}^{r}$ at $\left(s_{0}, D\right)$ is

$$
3 g-3+n-\text { (the number of } E_{j, k} \text { which are linearly independent). }
$$

Consider the coefficient of $b_{m}$ in $E_{j, k}$. This coefficient will be a linear combination of certain of the $\tau_{P_{j}, \nu}^{\prime}\left(Q_{m}\right) \zeta_{k}^{\prime}\left(Q_{m}\right)$. That is, the coefficient of $b_{m}$ will be a certain quadratic differential (the above linear combination of certain of the $d \tau_{P_{j}, \nu} d \zeta_{k}$ ) evaluated at the point $Q_{m}$. It should be noted that, by the symmetry of the matrix $M$ in the $b_{m}$, this quadratic differential does not depend on $m$, but only on $j$ and $k$. The coefficient of $b_{1}$ in $E_{j, k}$ is the value of this quadratic differential at $Q_{1}$, the coefficient of $b_{2}$ in $E_{j, k}$ the value at $Q_{2}$, etc. Put $\alpha_{j, k}$ equal to the above linear combination of certain of the $d \tau_{P_{j}, \nu} d \zeta_{k}$. Then $\alpha_{j, k}$ is a (not necessarily finite) quadratic differential.

Notation. Choose a local parameter $u_{m}$ on $X$ at $Q_{m}$ and write $\alpha_{j, k}=$ $g\left(u_{m}\right) d u_{m}^{2}$. Then we will write $\alpha_{j, k}\left(Q_{m}\right)$ for $g(0)$. Hence, by the above discussion, $\alpha_{j, k}\left(Q_{m}\right)$ is the coefficient of $b_{m}$ in $E_{j, k}$.

Our aim now is to show that, in certain situations, by suitably choosing the point $\left(Q_{1}, \cdots, Q_{3 g-3}\right)$, we may conclude that the $E_{j, k}$ are linearly independent. Assume that $r i \leqslant 3 g-3$. By elementary linear algebra, to conclude that the $E_{j, k}$ are linearly independent, it is sufficient to show that the matrix of coefficients

$$
A=\left[\alpha_{j, k}\left(Q_{m}\right)\right], \quad j=1, \cdots, r ; k=1, \cdots, i ; m=1, \cdots, r,
$$

is nonsingular. 
Lemma 4. Assume that the $\alpha_{j, k}$ for $j=1, \cdots, r$ and $k=1, \cdots, i$, are linearly independent and that $r i \leqslant 3 g-3$. Then we may choose a point $\left(Q_{1}, \cdots, Q_{3 g-3}\right)$ from the open set in $X^{3 g-3}$ in Theorem 2 such that each $Q_{m}$ is different from $P_{0}$ and no $Q_{m}$ is a zero of $d \zeta_{1}, \cdots, d \zeta_{g}$ and such that the above matrix $A$ is nonsingular.

Proof. The lemma will follow readily from the following

Sublemma. Let $\beta_{1}, \cdots, \beta_{n}$ be $n$ linearly independent quadratic differentials on $X$. Let $U$ be an open set contained in $X^{n}$. Then we may choose a point $\left(P_{1}, \cdots, P_{n}\right) \in U$ such that each $P_{m}$ is different from a finite set of points of $X$ and such that the matrix $\left[\beta_{j}\left(P_{k}\right)\right](j=1, \cdots, n, k=1, \cdots, n)$ is nonsingular.

Proof. By induction on $n$. If $n=1$, then $\beta_{1}$ is a nontrivial quadratic differential. Hence, $\beta_{1}$ is nonzero and finite on a dense open set of $X$. So, given any open set in $X$, there exists a point in that set satisfying the requirements of the Sublemma.

Now suppose $U$ is an open set contained in $X^{n}$. Let $V$ be the projection of $U$ onto $X^{n-1}$. Then $V$ is open and, by induction, we may choose a point $\left(P_{0}, \cdots, P_{n-1}\right) \in V$ such that each $P_{m}$ is different from a finite set of points of $X$ and such that the leading subdeterminant of order $n-1$ of the determinant

$$
\left|\begin{array}{ccc}
\beta_{1}\left(P_{1}\right) \cdots \beta_{1}\left(P_{n-1}\right) & \beta_{1} \\
\cdot & & \cdot \\
\cdot & & \cdot \\
\beta_{n}\left(P_{1}\right) \cdots \beta_{n}\left(P_{n-1}\right) & \beta_{n}
\end{array}\right|
$$

is nonzero. Expanding the full determinant by the last column, we obtain a nontrivial linear combination of $\beta_{1}, \cdots, \beta_{n}$. By the linear independence of these quadratic differentials, this linear combination is a nontrivial quadratic differential, hence is nonzero and finite on an open dense set $W$ contained in $X$. Since $U$ is open in $X^{n}$ and $W$ is dense in $X$, we may choose a point in the intersection of $U$ and $\left\{\left(P_{0}, \cdots, P_{n-1}\right)\right\} \times W$ which satisfies the requirements of our Sublemma.

Now, since the set of points in $X^{38-3}$ in Theorem 2 is open, it is easy to see that we may choose a point $\left(Q_{1}, \cdots, Q_{3 g-3}\right)$ in this set such that each $Q_{m}$ is different from $P_{0}$ and the zeros of $d \zeta_{1}, \cdots, d \zeta_{g}$ and so that $Q_{1}, \cdots$, $Q_{r i}$ make the matrix $A$ nonsingular. This completes the proof of the lemma.

We then have 
Proposition 12. Suppose $D$ is in $G_{n}^{r}(X)-G_{n}^{r+1}(X)$ and that $r i \leqslant$ $3 g-3$. Then if all the $\alpha_{j, k}$ are linearly independent, the dimension of the tangent space to $G_{n}^{r}$ at $\left(s_{0}, D\right)$ is $3 g-3+\tau+r$.

Proof. By Lemma 4 , we may choose a point $\left(Q_{1}, \cdots, Q_{3 g-3}\right)$ from the open set in Theorem 2 such that each of the $Q_{m}$ is different from $P_{0}$ and the zeros of $d \zeta_{1}, \cdots, d \zeta_{g}$ (note that this latter set includes the points of $D$ ), and such that the equations $E_{j, k}$ are linearly independent. Thus the dimension of the tangent space to $G_{n}^{r}$ at $\left(s_{0}, D\right)$ is $3 g-3+n-i r=3 g-3+\tau+r$.

In the next section, we show that if $D$ is in $G_{n}^{1}(X)-G_{n}^{2}(X)$, then the $\alpha_{j, k}$ are linearly independent. (Note that we have $i<3 g-3$ if $g>1$.)

5. The dimension of $G_{n}^{1}-G_{n}^{2}$. For simplicity, we will first treat a divisor consisting of $n$ distinct points. So assume $D=P_{1}+P_{2}+\cdots+P_{n}$, all points distinct, is in $G_{n}^{1}(X)-G_{n}^{2}(X)$. Recall that the matrix $M$ is

$$
M=\left[\begin{array}{lll}
\varphi_{i, k}\left(P_{j}\right) & \vdots \epsilon\left(s_{j} \varphi_{j, k}^{\prime}\left(P_{j}\right)+\sum_{m=1}^{3 g-3} b_{m} \tau_{P_{j}, 0}^{\prime}\left(Q_{m}\right) \xi_{k}^{\prime}\left(Q_{m}\right)\right) \\
j=1, \cdots, n & \vdots & j=1, \cdots, n \\
k=1, \cdots, g-i & k=g-i+1, \cdots, g
\end{array}\right] .
$$

Let $|\hat{j}|$ denote the minor of order $n-1$ obtained by omitting the $j$ th row from the matrix $\left[\varphi_{j, k}\left(P_{j}\right)\right](j=1, \cdots, n, k=1, \cdots, g-i)$. Then we have

$$
\alpha_{1, k}(Q)=\sum_{j=1}^{n}(-1)^{j-1}|\hat{j}| \tau_{P_{j}, 0}^{\prime}(Q) \xi_{n+k-1}^{\prime}(Q)
$$

for $k=1,2, \cdots, i$. Suppose we had a linear relation of the form $\sum_{k=1}^{i} a_{k} \alpha_{1, k}$ $=0$ with some $a_{l}$ nonzero. Then this would imply that

$$
\left(\sum_{j=1}^{n}(-1)^{j-i}|\hat{j}| \tau_{P_{j}, 0}^{\prime}(Q)\right)\left(\sum_{k=1}^{i} a_{k} \zeta_{n+k-1}^{\prime}(Q)\right)=0 \text {. }
$$

But the $d \tau_{P_{j}, 0}, j=1, \cdots, n$, are linearly independent, since they have poles at different points. This, together with the fact that $|\hat{n}| \neq 0$, implies that there is a dense open set of points of $X$ where the expression $\sum_{j=1}^{n}(-1)^{j-1}|\hat{j}| \tau_{p_{j}, 0}^{\prime}(Q)$ is nonzero.

And the linear independence of $d \zeta_{n}, \cdots, d \zeta_{g}$, together with the fact that some $a_{l}$ is nonzero, implies that the expression $\Sigma_{k=1}^{i} a_{k} \zeta_{n+k-1}^{\prime}(Q)$ is nonzero on a dense open set of points of $X$. Hence, we may choose a point $Q$ such that (*) is nonzero, contradicting the assumption that $\alpha_{1,1}, \cdots, \alpha_{1, i}$ are linearly dependent. 
Now suppose $D=m_{1} P_{1}+\cdots+m_{d} P_{d}$ is in $G_{n}^{1}(X)-G_{n}^{2}(X)$. Then we have

$$
\alpha_{1, k}(Q)=\zeta_{n+k-1}^{\prime}(Q)\left(|\hat{1}| \tau_{P_{1}, 0}^{\prime}(Q)+\cdots+(-1)^{n-1} \hat{\mid n} \mid \tau_{P_{d}, m_{d^{-1}}}^{\prime}(Q)\right) .
$$

Hence, if there existed a linear relation $\Sigma_{k=1}^{i} a_{k} \alpha_{1, k}=0$, we would have

$$
\left(\sum_{k=1}^{i} a_{k} \xi_{n+k-1}^{\prime}(Q)\right)\left(|\hat{1}| \tau_{P_{1}, 0}^{\prime}(Q)+\cdots+(-1)^{n-1}|\hat{n}| \tau_{P_{d} m_{d^{-1}}}^{\prime}(Q)\right)=0 \text {. }
$$

The same reasoning as in the case of simple points applies, since $d \tau_{P_{1}, 0}$, $\cdots, d \tau_{P_{d}, m_{d^{-1}}}$ are easily seen to be linearly independent (they either have poles at different points or have poles of differing orders at the same point).

REMARK. The above reasoning shows that if $D \in G_{n}^{r}-G_{n}^{r+1}$, then the $\alpha_{j, k}$ for a fixed $j$ are linearly independent.

THEOREM 3. $G_{n}^{1}-G_{n}^{2}$, if nonempty, is smooth of pure dimension $3 g-$ $3+\tau+1$.

Proof. Let $\left(s_{0}, D\right)$ be any point of $G_{n}^{1}-G_{n}^{2}$. By Proposition 12 and the work of this section, we may conclude that the dimension of the tangent space to $G_{n}^{1}$ at $\left(s_{0}, D\right)$ is $3 g-3+\tau+1$. By Proposition 4 , the dimension of $G_{n}^{1}$ at $\left(s_{0}, D\right)$ is at least $3 g-3+\tau+1$, hence $G_{n}^{1}$ is smooth at $\left(s_{0}, D\right)$ and has dimension precisely $3 g-3+\tau+1$.

REMARK. Theorem 3 does not depend upon $\tau$ being nonnegative.

THEOREM 4. Suppose that $G_{n}^{1}(X)-G_{n}^{2}(X)$ is nonempty for a generic curve $X$. Then $G_{n}^{1}(X)-G_{n}^{2}(X)$, for a generic $X$, is smooth of pure dimension $\tau+1$.

Proof. Under our assumption, the image of $G_{n}^{1}-G_{n}^{2}$ in $T_{g}$ would be a dense open subspace $U$. By Sard's Theorem, since $G_{n}^{1}-G_{n}^{2}$ is smooth, the generic fiber of the map $G_{n}^{1}-G_{n}^{2} \rightarrow U$ is smooth. And since $U$ has dimension $3 g-3$ and $G_{n}^{1}-G_{n}^{2}$ has dimension $3 g-3+\tau+1$, the generic fiber has dimension $\tau+1$. Thus, for a generic curve, $G_{n}^{1}(X)-G_{n}^{2}(X)$ is smooth of dimension $\tau+1$.

REMARK. If $\tau \geqslant 0$, then by [10] we know that $G_{n}^{r}(X)$ is nonempty. If we knew that $G_{n}^{r}(X)$ were reduced for a generic $X$, then, since the points of $G_{n}^{r+1}$ are singular points of $G_{n}^{r}$, we could conclude that $G_{n}^{r}(X)-G_{n}^{r+1}(X)$ is nonempty for generic $X$ if $\tau \geqslant 0$.

6. Moduli of trigonal curves. A trigonal curve is a curve $X$ such that $G_{3}^{1}(X)$ is nonempty. We can use Theorem 3 to compute the moduli of trigonal curves. By Clifford's Theorem, $G_{3}^{2}$ is empty hence, by Theorem 3, $G_{3}^{1}$, if 
nonempty, is smooth of pure dimension $3 g-3+\tau+1$. Now $\tau=2(3-1)-$ $g=4-g$, so $G_{3}^{1}$, if nonempty, has dimension $2 g+2$.

By Theorem 1 of [12], we have that, for $g \geqslant 4$, if $G_{3}^{1}(X)$ is nonempty, then every component has dimension at least $5-g$ and at most 2 , with the upper bound occurring if and only if $X$ is hyperelliptic. So, if there exists a nonhyperelliptic trigonal curve of genus $g$, then we must have that the dimension of the generic fiber of the map $G_{3}^{1} \rightarrow T_{g}$ is 1. Examples of such curves (for every $g \geqslant 3)$ are given in [1a, p. 196].$\left(^{1}\right)$ Hence, the dimension of the subvariety of $T_{g}$, for $g \geqslant 4$, of trigonal curves is $2 g+2-1=2 g+1$. This agrees with the number which appears in Segre [20] and Severi [22].

\section{BIBLIOGRAPHY}

1. A. Andreotti, On a theorem of Torelli, Amer. J. Math. 80 (1958), 801-828. MR 21 \#1309.

1a. A. Andreotti and A. L. Mayer, On period relations for abelian integrals on algebraic curves, Ann. Scuola Norm. Sup. Pisa (3) 21 (1967), 189-238. MR 36 \#3792.

2. A. Brill and M. Noether, Über die algebraischen Funktionen und ihre Anwendung in der Geometrie, Math. Ann. 7 (1874).

3. H. M. Farkas, Special divisors and analytic subloci of the Teichmueller space, Amer. J. Math. 88 (1966), 881-901. MR 35 \#4406.

4. G. Grauert, Ein Theorem der analytischen Garbentheorie und die Modulrdume komplexer Strukturen, Inst. Hautes Études Sci. Publ. Math. No. 5 (1960). MR 22 \#12544. Paris.

5. A. Grothendieck, Exposés in Séminaire Cartan, 1960/61, Secrétariat mathématique,

6. R. C. Gunning, Jacobi varieties, Princeton Univ. Press, Princeton, N. J., 19/12.

7. B. Iversen, Linear determinants with applications to the Picard scheme of a family of algebraic curves, Lecture Notes in Math., vol. 174, Springer-Verlag, Berlin, 1970. MR 45 \#1917.

8. G. Kempf, Schubert methods with an application to algebraic curves, Stichting Mathematisch Centrum, Amsterdam, 1971.

9. G. Kempf and D. Laksov, The determinantal formula of Schubert calculus, Acta Math. 132 (1974), 153-162.

10. S. L. Kleiman and D. Laksov, On the existence of special divisions, Amer. J. Math. 94 (1972), 431-436.

11. Another proof of the existence of special divisors, Acta Math. 132 (1974), 163-176.

12. H. H. Martens, On the varieties of special divisors on a curve. I, J. Reine Angew. Math. 227 (1967), 111-120. MR 35 \#6682.

13. - Varieties of special divisors on a curve. II, J. Reine Angew. Math. 233 (1968), 89-100. MR 39 \#2760.

14. A. Mattuck and A. Mayer, The Riemann-Roch theorem for algebraic curves, Ann. Scuola Norm. Sup. Pisa (3) 17 (1963), 223-237. MR 29 \#102.

15. A. Mayer, Rauch's variational formula and the heat equation, Math. Ann. 181 (1969), 53-59. MR 40 \#1588.

16. T. Meis, Die minimale Bldtterzahl der Konkretisierung einer kompakten Riemannschen Fläche, Schr. Math. Inst. Univ. Munster No. 16 (1960). MR 26 \#5158.

17. C. Patt, Variations of Teichmuller and Torelli surfaces, J. Analyse Math. 11 (1963), 221-247. MR $28 \# 4103$.

18. H. E. Rauch, Weierstrass points, branch points and moduli of Riemann surfaces, Comm. Pure Appl. Math. 12 (1959), 543-560. MR 22 \#1666.

(1) The author wishes to thank the referee for pointing out this reference. 
19. M. Schiffer and D. C. Spencer, Functionals of finite Riemann surfaces, Princeton Univ. Press, Princeton, N. J., 1964.

20. B. Segre, Sui moduli delle curve poligonali $\cdots$, Math. Ann. 100 (1928), 537551.

21. F. Severi and E. Löffler, Vorlesungen über algebraischen Geometrie, Teubner, Leipzig, 1921.

22. - Sul teorema di esistenza di Riemann, Rend. Circ. Mat. Palermo 46 (1922).

23. G. Springer, Introduction to Riemann surfaces, Addison-Wesley, Reading, Mass., 1957. MR 19, 1169.

DEPARTMENT OF MATHEMATICS, LOUISIANA STATE UNIVERSITY, BATON ROUGE, LOUISIANA 70803 